\title{
Prostatic fluid exosome-mediated microRNA-155 promotes the pathogenesis of type IIIA chronic prostatitis
}

\author{
Baixiong Zhao ${ }^{1}$, Jun Zheng ${ }^{1}$, Yang Qiao ${ }^{1}$, Yongquan Wang ${ }^{1}$, Yang Luo ${ }^{2}$, Dinglin Zhang ${ }^{1,3}$, Qiyan Cai ${ }^{4}$, \\ Yang $\mathrm{Xu}^{4,5}$, Zhansong Zhou ${ }^{1}$, Wenhao Shen ${ }^{1}$ \\ ${ }^{1}$ Department of Urology, Southwest Hospital, The First Affiliated Hospital of Third Military Medical University (Army Medical University), \\ Chongqing, China; ${ }^{2}$ Center of Smart Laboratory and Molecular Medicine, Medical College, Chongqing University, Chongqing, China; ${ }^{3}$ Department \\ of Chemistry, College of Basic Medicine, Third Military Medical University (Army Medical University), Chongqing, China; ${ }^{4}$ Department of \\ Histology and Embryology, College of Basic Medicine, Army Medical University, Chongqing, China; ${ }^{5}$ Department of Obstetrics and Gynecology, \\ The Second Affiliated Hospital of Army Medical University, Chongqing, China \\ Contributions: (I) Conception and design: W Shen, B Zhao; (II) Administrative support: W Shen, B Zhao; (III) Provision of study materials or \\ patients: W Shen, Z Zhou; (IV) Collection and assembly of data: Y Qiao, J Zheng; (V) Data analysis and interpretation: B Zhao; (VI) Manuscript \\ writing: All authors; (VII) Final approval of manuscript: All authors. \\ Correspondence to: Zhansong Zhou; Wenhao Shen. Department of Urology, Southwest Hospital, The First Affiliated Hospital of Third Military \\ Medical University (Army Medical University), Chongqing 400038, China. Email: zhouzhansong@sohu.com; chongqingswh@aliyun.com.
}

Background: The latest research has shown that exosomes play an important role in cell-to-cell communication and are closely related to the occurrence of many chronic inflammatory diseases. However, no studies have clarified whether exosomes are involved in the pathogenesis of aseptic inflammation, type IIIA chronic prostatitis/chronic pelvic pain syndrome (CP/CPPS-A). This study aimed to explore the relationship between prostatic fluid exosomes and CP/CPPS-A and reveal new pathogenesis.

Methods: Our group collected prostatic fluid samples from CP/CPPS-A patients and normal adult men. Electron microscope, quantitative PCR (qPCR), Western Blot, nanoparticle tracking analysis, hematoxylinand-eosin (HE) staining, immunofluorescence staining and miRNA-155 functional analysis were used to verify the role of exosomes in CP/CPPS-A in vivo and in vitro.

Results: Exosomes were abundantly enriched in the prostatic fluid of CP/CPPS-A patients and selectively overloaded with microRNA-155 (miRNA-155). These exosomes were taken up by prostatic stromal cells in large quantities. They activated interleukin (IL)- 8 and tumor necrosis factor-alpha (TNF- $\alpha$ ) expression in vitro, and the integrity of the exosomes' plasma membrane is a necessary condition for information transmission by exosomes. In in vivo experiments, histological results showed that prostatic fluid exosomes induced prostatitis in rats. Also, immunofluorescence staining showed excessive activation of IL- 8 , TNF- $\alpha$, and inducible nitric oxide synthase (iNOS).

Conclusions: Exosomes in the prostatic fluid and the miRNA-155 contained therein were may be involved with the pathogenesis of CP/CPPS-A.

Keywords: Type IIIA chronic prostatitis; exosomes; microRNA-155 (miRNA-155)

Submitted Feb 13, 2021. Accepted for publication Mar 19, 2021.

doi: $10.21037 /$ tau-21-139

View this article at: http://dx.doi.org/10.21037/tau-21-139

\section{Introduction}

Prostatitis is a common disease of the urinary system. Symptoms caused by prostatitis, such as pain, abnormal urination, and sexual dysfunction, seriously affect quality of life (1). Type III chronic prostatitis, a type of prostatitis known as chronic prostatitis/chronic pelvic pain syndrome (CP/CPPS), accounts for approximately $90 \%$ of prostatitis cases, with an overall incidence in the population of 
approximately $9-15 \%(2,3)$. CP/CPPS is a non-bacterial disease and is divided into the more common type-A (nonbacterial inflammatory) and type-B (non-bacterial noninflammatory) categories according to the white blood cell count in prostatic fluid (4). Because of the particular features of CP/CPPS-A, definitive etiology studies have failed to clarify its pathogenesis. A new entry point is urgently needed in clinical practice to promote further research of CP/CPPS-A.

Exosomes are small vesicles of approximately 30 $200 \mathrm{~nm}$ in diameter that are generated from cellular endosomes with a double-layer plasma membrane structure and through exocytosis are released by cells into the extracellular space or biological fluids. Exosomes play an important role in cell-to-cell communication (5-7) and are secreted by many cell types, including epithelial and tumor cells $(8,9)$. Exosomes contain abundant lipids, proteins, and microRNAs (miRNAs) from maternal cells and other signaling molecules (10). These substances migrate with exosomes to the recipient cells, thereby regulating the recipient cells' biological states (11). Recent evidence has shown that exosomes are closely related to the occurrence of chronic inflammatory diseases. For example, hepatocytes infected with hepatitis B viruses secreted many exosomes rich in $m i R N A-122$. These exosomes were transferred to monocytes to induce the synthesis of interleukin (IL)-1 $\beta$ and tumor necrosis factor-alpha (TNF- $\alpha$ ). The exosomes secreted by neutrophils deliver $\operatorname{miRNA-155}$ to vascular endothelial cells to initiate atherosclerosis and promote chronic vascular inflammation (12-15).

MiRNAs are endogenous non-coding RNA molecules that regulate the expression of downstream genes by inhibiting translation or degrading target mRNAs $(16,17)$. They are involved in many physiological processes, such as cell proliferation, cell differentiation, apoptosis, tissue, and organ development, and play a key role in the occurrence and progression of inflammation (18-20). For example, miRNA-155 promotes inflammatory cytokines and nitric oxide production to regulate the immune response mediated by microglial cells (21); miRNA-155 is selectively introduced into macrophages to activate TNF- $\alpha$ and IL-6 production, thereby promoting the inflammatory response of lung tissue (22). Importantly, a previous highthroughput sequencing study has shown that miRNA-155 is significantly increased in the prostatic fluid of patients with chronic prostatitis (23).

Prostate epithelial cells and prostatic stromal cells are two major cell types in prostate tissue and are adjacent in histological position. A study has shown that prostatic stromal cells are immunogenic, and they secrete cytokines, such as IL-8, under certain stimulating conditions, promote chemotaxis of neutrophils, and regulate the local immune response (24). Also, the prostatic fluid is a special body fluid of great significance to prostatitis diagnosis that is mainly secreted by prostate epithelial cells. However, no reports on the influence of exosomes and their prostatic fluid contents on the occurrence and progression of prostatitis are available. On this basis, this study explored the role of prostatic fluid-derived exosomes and their contained $m i R N A-155$ in the pathogenesis of type IIIA chronic prostatitis. We present the following article in accordance with the MDAR reporting checklist (available at http:// dx.doi.org/10.21037/tau-21-139).

\section{Methods}

\section{Isolation, identification, and characterization of prostatic fluid exosomes}

\section{Collection of prostatic fluid}

The seventeen prostatic fluid samples from CP/CPPS-A patients were from patients from the Department of Urology, the First Affiliated Hospital of Army Medical University (Chongqing, China), and Eight volunteers included in the study provided written informed consent before participating in this study healthy adult males were from people randomly recruited from the population. All volunteers included in the study provided written informed consent before participating in this study. The research protocol was reviewed and approved by the Ethics Committee of the First Affiliated Hospital of Army Medical University (Ethics approval number: KY201801). The study was conducted in accordance with the Declaration of Helsinki (as revised in 2013).

Inclusion criteria for patients with CP/CPPS-A were: (I) males between 18 and 55 years old; (II) typical clinical manifestation of chronic prostatitis lasting more than three months; (III) with >10 white blood cells per high-power microscope in routine microscopic examination of prostatic fluid; and (IV) with negative results for common bacteria in urine culture before and after prostate massage. Exclusion criteria for patients with CP/CPPS-A were patients with varicocele, urinary tract infection, urinary tract tumor(s), other diseases in the urinary system, or other systemic diseases. Inclusion criteria for healthy adult males were: (I) 1.18 to 55 years old; (II) without clinical symptoms related 
to chronic prostatitis in the past one year; (III) with normal prostatic fluid in routine microscopic examination; and (IV) with negative results of common bacteria in urine culture before and after prostate massage, and the exclusion criteria were individuals having diseases in the urinary system or other systemic diseases.

\section{Isolation of prostatic fluid exosomes}

The prostatic fluid sample from each individual was collected in a $1.5-\mathrm{mL}$ sterile Eppendorf tube as described in the literature (25), followed by ultracentrifugation at $3,000 \mathrm{~g}$ and $4{ }^{\circ} \mathrm{C}$ for $10 \mathrm{~min}$ to collect the supernatant and a dilution with an equal volume of sterile phosphate-buffered saline (PBS, $\mathrm{pH}$ 7.4). The diluted supernatant was further centrifuged at $10,000 \mathrm{~g}$ and $4{ }^{\circ} \mathrm{C}$ for $30 \mathrm{~min}$, followed by collecting and filtering the supernatant with a $0.22-\mathrm{mm}$ filter (Millipore, Burlington, MA). The filtered supernatant was further centrifuged at $150,000 \mathrm{~g}$ and $4{ }^{\circ} \mathrm{C}$ for $2 \mathrm{~h}$ in a CP70ME ultracentrifuge (Hitachi, Japan). To further purify the exosomes, the pellet was washed with a large amount of sterile PBS, centrifuged at $150,000 \mathrm{~g}$ and $4{ }^{\circ} \mathrm{C}$ for $1 \mathrm{~h}$ to collect the precipitate, and resuspended in $100 \mu \mathrm{L}$ sterile PBS for subsequent experiments.

\section{Identification and characterization of prostatic fluid exosomes}

A $30 \mu \mathrm{L}$ exosome sample was placed on a copper mesh and subjected to negative staining at room temperature before observation under an electron microscope (JEOL, Japan). The isolated exosomes were subjected to nanoparticle tracking analysis (NTA) using the NanoSight NS300 instrument (Malvern, UK). Protein markers CD9 and CD63 were used for the characterization of exosomes by Western blot analysis. After collecting the protein samples quantified by Pierce BCA protein assay kit (Thermo Fisher Scientific), the protein samples were mixed with $5 \times$ SDSPAGE loading buffer and incubated at $95{ }^{\circ} \mathrm{C}$ for $5 \mathrm{~min}$. After cooling, the sample mixtures were centrifuged at $10,000 \mathrm{~g}$ for $1 \mathrm{~min}$ to collect the supernatants, which were separated in $15 \%$ SDS-PAGE gel $(80 \mathrm{~V}$ in the concentrated gel for $30 \mathrm{~min}$; $120 \mathrm{~V}$ in separating gel for $60 \mathrm{~min}$ ), followed by transferring the separated proteins to polyvinylidene fluoride or polyvinylidene difluoride (PVDF) membranes. The protein membranes were blocked in a buffer containing $5 \%$ skim milk powder and incubated at room temperature on a shaker for $2 \mathrm{~h}$. The protein membranes were then incubated with primary antibodies (1:1,000 anti-CD 9 and 1:1,000 anti-CD63, Thermo Fisher Scientific; 1:1,000 anti-
GAPDH, Santa Cruz, Santa Cruz, CA) independently at $4{ }^{\circ} \mathrm{C}$ overnight. After washing in Tris-buffered saline containing $0.5 \%$ TWEEN 20 (0.5\% TBST solution) three times, the PVDF membranes were incubated with the corresponding secondary antibodies $(1: 2,000$, Cell Signaling Technology, Danvers, MA), which contained 5\% skim milk powder at room temperature for $2 \mathrm{~h}$ on a shaker. The PVDF membranes were rinsed three times with $0.5 \%$ TBST solution (10 min each) and subsequently developed with the enhanced chemiluminescent (ECL) substrate kit (Amersham Biosciences, Piscataway, NJ) before analyzing the results. The GADPH protein was used as an internal reference control for Western blotting analysis.

The exosomal proteins were quantified by the bicinchoninic acid (BCA) assay, followed by the addition of $10 \mu \mathrm{L}$ exosome sample with $100 \mu \mathrm{L}$ RIPA buffer and $1 \mu \mathrm{L}$ PMSF in a sterile Eppendorf tube, a mixing step, and incubation on ice for $30 \mathrm{~min}$ before centrifugation at $12,000 \mathrm{~g}$ and $4{ }^{\circ} \mathrm{C}$ for $5 \mathrm{~min}$. According to the manufacturer instructions, the collected supernatant was subjected to exosomal protein quantification using a BCA kit (Beyotime Biotechnology, Shanghai, China). The ExoELISA-ULTRA Complete Kit (CD63 detection) (System Biosciences, Palo Alto, CA) was used to detect exosome concentration by adding $20 \mu \mathrm{L}$ exosome samples with $100 \mu \mathrm{L}$ coating buffer according to the manufacturer instructions.

\section{Tracing of exosomes and detection of miRNA-155 in exosomes}

\section{Cell culture and tracing of exosomes}

Normal human prostatic stromal cell line (WPMY-1) purchased from the American Type Culture Collection (Manassas, VA) was placed in Dulbecco's Modified Eagle Medium (DMEM) containing 10\% fetal bovine serum (Invitrogen, Carlsbad, CA) and incubated at $37^{\circ} \mathrm{C}$ in an incubator with $5 \% \mathrm{CO}_{2}$, with medium change every $48 \mathrm{~h}$. The cells were sub-cultured when they reached $80-90 \%$ confluency.

The exosomes were labeled using PKH26 red fluorescent labeling kit (Sigma-Aldrich, St. Louis, MO) according to the manufacturer instructions, followed by co-culturing exosomes with WPMY-1 cells for $12 \mathrm{~h}$ and performing DAPI nuclear staining on the WPMY-1 cells. Then, $500 \mu \mathrm{L}$ $\mathrm{DiO}$ cell-labeling solution (Beyotime Biotechnology) was used to label the plasma membrane at $37^{\circ} \mathrm{C}$ in the dark for $1 \mathrm{~h}$, followed by rinsing with sterile PBS and observation under a confocal microscope (Olympus, Japan). 
Total RNA extraction and quantitative PCR (qPCR) analysis of miRNA-155 concentration

The total RNA of the cells was extracted using TRIzol reagent (Invitrogen) and the Hairpin-it miRNA qPCR Quantitation Kit (GenePharma, Shanghai, China) was used to synthesize cDNA by reverse transcription. Subsequently, the PowerUp SYBR Master Mix (Thermo Fisher Scientific, Waltham, MA) was used for qPCR analysis in the StepOne Plus Real-Time PCR System (Applied Biosystems, Waltham, MA) according to the manufacturer instructions. The glyceraldehyde 3-phosphate dehydrogenase $(G A P D H)$ was used as the internal reference for correction, and the target miRNA expression level was calculated using the comparative threshold cycle (CT) method $\left(2^{-\Delta \Delta C}\right)$. The primer sequences used for qPCR were: 5'-ACCCACTCCTCCACCTTTGA-3' for GAPDH-forward primer; 5'-CTGT TGCTGTAGCCAAATTCGT-3' for GADPHreverse primer; 5'-UUAAUGCUAAUCGUGAUAGGGGU-3' for $m i R N A-155$ primer.

\section{Destruction of exosomal membrane and evaluation of prostatitis in vitro}

\section{Ultrasound rupture of exosomal membrane}

An ultrasonic disruptor (Bioruptor, Diagenode, Liège, Belgium) was used to mechanically disrupt the exosomal membrane structure on ice for $5 \mathrm{~min}$. The cycle period was a $30 \mathrm{~s}$ cooling time every $10 \mathrm{~s}$ of ultrasonic operation.

\section{Detection of inflammatory cytokines}

We tested four groups: blank control (in vivo: NS—normal saline; in vitro: $\mathrm{PBS}$ ); normal control (NC-prostatic fluid exosomes of healthy individuals); CP/CPPS-A untreated (CP/ CPPS-A - prostatic fluid exosomes of CP/CPPS-A patients); and $\mathrm{CP} / \mathrm{CPPS}-\mathrm{A}$ plus ultrasound-rupture treatment $(\mathrm{CP} /$ CPPS-A + US-prostatic fluid exosomes of CP/CPPS-A patients with ultrasound-rupture treatment) groups. In in vitro experiments, each exosome group was co-co-cultured with WPMY- 1 cells at $37{ }^{\circ} \mathrm{C}$ and $5 \% \mathrm{CO}_{2}$. The supernatants were collected after $48 \mathrm{~h}$, followed by 1,000 rpm for $5 \mathrm{~min}$ to isolate the supernatant for detecting IL-8 and TNF- $\alpha$ contents using the corresponding ELISA kits (Absin, Shanghai, China) following the manufacturer instructions.

\section{Evaluation of prostatitis in animal experiments}

Experimental animals and prostatitis modeling Adult male Sprague-Dawley rats weighing $350 \pm 20 \mathrm{~g}$ were purchased from the Experimental Animal Center of Army Medical University. The research protocol of animal experiments was reviewed and approved by the Laboratory Animal Welfare and Ethics Committee of Army Medical University (Animal ethics lot number: AMUWEC2020939). After routine hair removal and skin disinfection of the lower abdomen and intraperitoneal injection of pentobarbital sodium for anesthesia, each rat underwent lower abdomen incision layer by layer under aseptic surgical conditions to expose the bladder and the prostate behind the bladder. A sterile micro-syringe was used to inject $5 \mu \mathrm{L}$ of extracted prostatic fluid-derived exosomes into the left and right ventral lobes of the prostate. The rats in the blank control group were injected with normal saline. Subsequently, the abdomen of each rat was closed layer by layer. All animals were bred routinely after waking up with free access to drinking water, euthanized a week later, and subjected to systemic perfusion and fixation with $4 \%$ paraformaldehyde.

\section{Prostate histopathology and immunofluorescence staining}

We performed histopathology on rat prostate tissue of different groups. The prostate tissue specimens were paraffin-embedded following the conventional method, followed by sectioning in 5 - $\mu$ m thick sections and hematoxylin-and-eosin (HE) staining. In the immunofluorescence staining, the sections were thoroughly washed with $0.01 \mathrm{~mol} / \mathrm{L}$ PBS and incubated in deionized water containing $3 \% \mathrm{H}_{2} \mathrm{O}_{2}$ for 10 min to block the endogenous peroxidase, followed by incubation with rabbit anti-IL-8 (1:200, Affinity Biosciences, Cincinnati, OH, USA), rabbit anti-iNOS (1:200 Affinity Biosciences), or goat anti-TNF- $\alpha$ (1:200, R\&D Systems, Minneapolis, $\mathrm{MN})$ at $4{ }^{\circ} \mathrm{C}$ overnight. After washing steps, the tissue sections were incubated with the corresponding secondary antibodies [TRITC goat anti-rabbit immunoglobulin G (1:100, Abcam) and FITC rabbit anti-goat immunoglobulin $\mathrm{G}(1: 100$, Abcam) $]$ at room temperature for $1 \mathrm{~h}$, followed by DAPI nuclear staining for $10 \mathrm{~min}$, mounting the slides, and observation under a fluorescence microscope (Zeiss, Germany).

\section{miRNA-155 functional analysis}

A day before cell transfection, the WPMY-1 cells were inoculated into a 24 -well plate containing $500 \mu \mathrm{L}$ culture medium until reaching $50-80 \%$ confluency. 
A

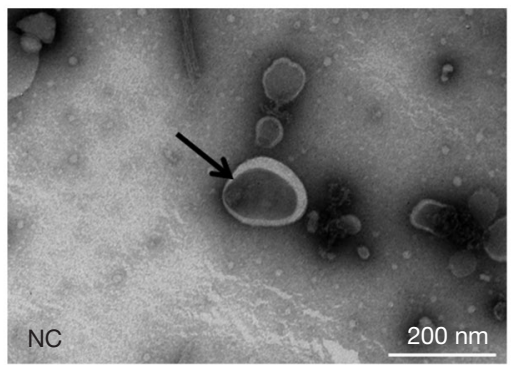

C
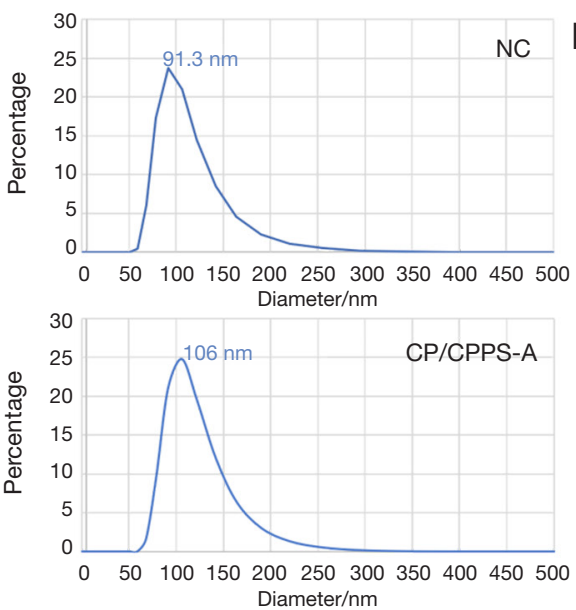

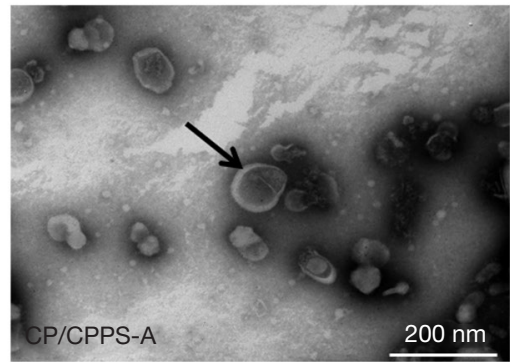

B

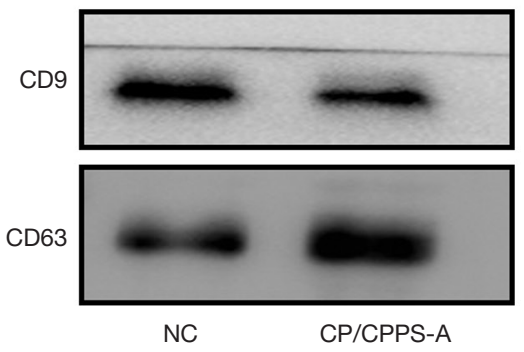

D

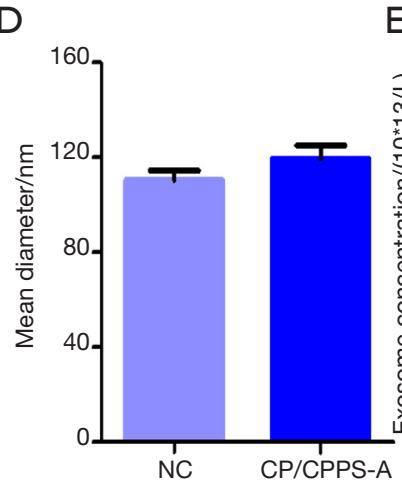

E

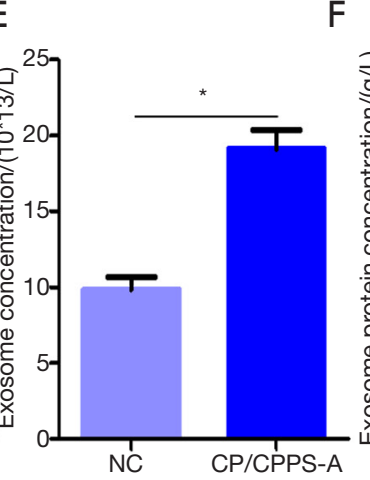

$\mathrm{F}$

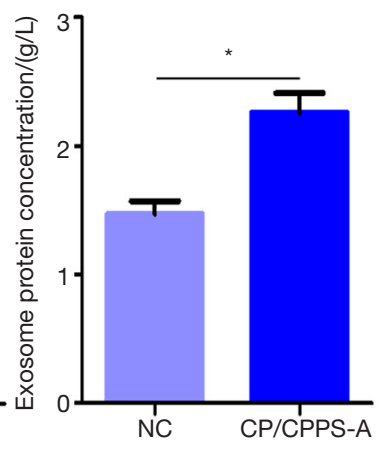

Figure 1 Isolation, identification, and characterization of prostatic fluid exosomes. (A) Electron transmission microscopy observed the prostatic fluid exosomes from a healthy individual and a CP/CPPS-A patient. Scale bar $=200 \mathrm{~nm}$. (B) Western blotting analysis of exosomal surface protein markers, CD9, and CD63. (C) Exosomal diameter distribution is shown by NTA. (D) The average size of exosomes derived from NTA. (E) Results of the concentration of exosomes in prostatic fluid. (F) Exosomal protein concentration measured by the BCA method. *, $\mathrm{P}<0.05$. NC, normal control; CP/CPPS-A, type IIIA chronic prostatitis/chronic pelvic pain syndrome.

Lipofectamine 2000 (Invitrogen) reagent was used according to the manufacturer's instructions to transfect the miRNA-155 mimics/inhibitor and NC miRNA-155 mimics/inhibitor (mirVana ${ }^{\mathrm{TM}}$, Invitrogen) into the WPMY-1, with mimics of $50 \mathrm{nM}$ and inhibitors of $100 \mathrm{~nm}$ in both. The effects were tested $24 \mathrm{~h}$ after transfection using qPCR and ELISA described as aforementioned.

\section{Statistical analysis}

Quantitative data were presented as a means \pm standard error of the mean (SEM). Comparisons of means were performed using Student's $t$-test or one-way ANOVA followed by Dunnett's post hoc test using SPSS 23.0 software (IBM SPSS, Armonk, NY). The threshold of statistical significance was set at $\mathrm{P}<0.05$.

\section{Results}

\section{Isolation, identification, and characterization of buman prostatic fluid exosomes}

This study successfully isolated exosomes from human prostatic fluid by ultracentrifugation and observed them by electron microscopy. Transmission electron microscopy showed that exosomal vesicles had a typical double-layer plasma membrane structure, elliptical or circular, with a diameter of approximately 40-150 nm (Figure 1A). Western blotting analysis for detecting surface protein markers of exosomes showed positive CD9 and CD63 expression (Figure 1B), which further proved the successful isolation of exosomes in this study.

To further study the characteristics of the isolated exosomes, NTA showed no significant difference in the peak or diameters of prostatic fluid exosomes between 


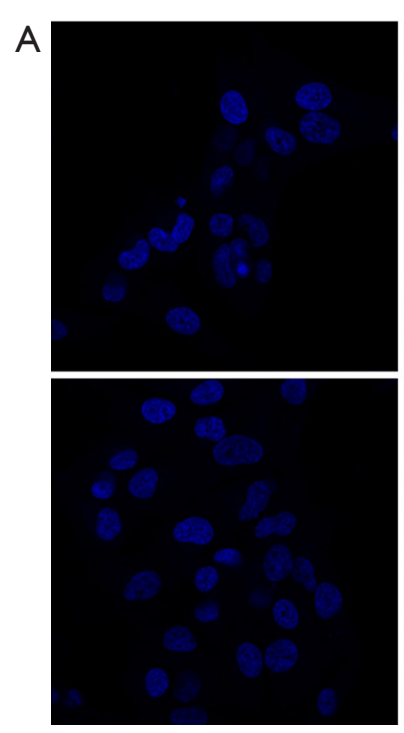

DAPI
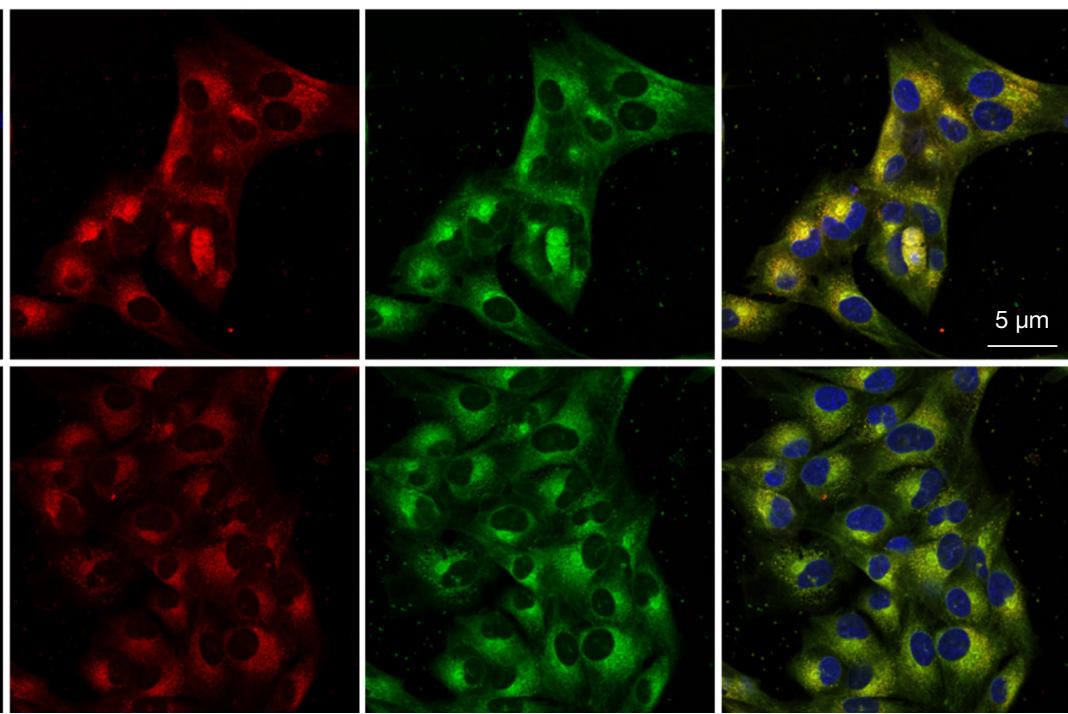

$\mathrm{DiO}$

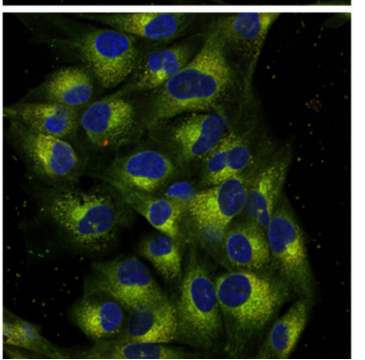

Merge

C

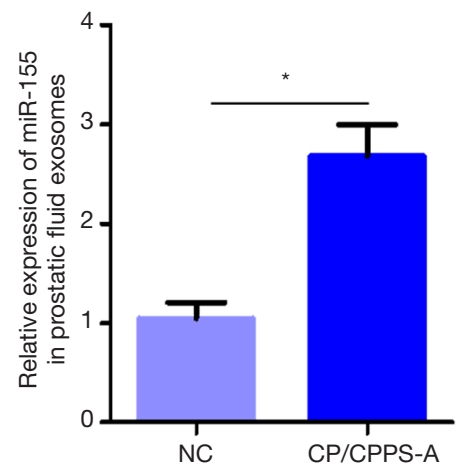

Figure 2 Exosomes were selectively loaded with miRNA-155 and taken up by prostatic stromal cells. (A) The prostatic fluid exosomes of the healthy individual and CP/CPPS-A patient marked by PKH26 were significantly engulfed and taken up by the prostatic stromal cells. (B) The relative miRNA-155 expression in NC's prostatic fluid and the CP/CPPS-A groups measured by qPCR. (C) The relative miRNA-155 expression in prostatic fluid exosomes of the NC and the CP/CPPS-A groups measured by qPCR. *, P<0.05. NC, normal control; CP/ CPPS-A, type IIIA chronic prostatitis/chronic pelvic pain syndrome.

healthy individuals and CP/CPPS-A patients (Figure 1C,D). However, the detection of exosome concentrations showed that the exosome concentration in CP/CPPS-A patients was significantly higher than that of healthy individuals (Figure 1E). Similarly, the protein concentrations of exosomes in the CP/CPPS-A patients were also significantly higher than those in healthy individuals (Figure $1 F$ ), suggesting that exosomes were excessively secreted in the prostatic fluid of the CP/CPPS-A patients.

\section{Prostatic fluid exosomes transport miRNA-155 to the prostatic stromal cells}

To clarify whether exosomes in prostatic fluid were taken up by the WPMY-1 cells, we used PKH26 dye to label the exosomes in red and then co-cultured them with WPMY1 human prostatic stromal cells, followed by the addition of DiO and DAPI to label the cell membranes and nuclei, respectively. Confocal microscopy showed that exosomes successfully entered the WPMY-1 cells, confirming that prostatic fluid exosomes were engulfed by the prostatic stromal cells in large amounts (Figure 2A).

We also tested the concentrations of miRNA-155 in prostatic fluid and exosomes in different groups. Our results showed that the concentrations of miRNA-155 in the prostatic fluid and exosomes of CP/CPPS-A patients (CP/CPPS-A group) were significantly higher than in the healthy individuals (NS group) (Figure 2B and 2C), 
A

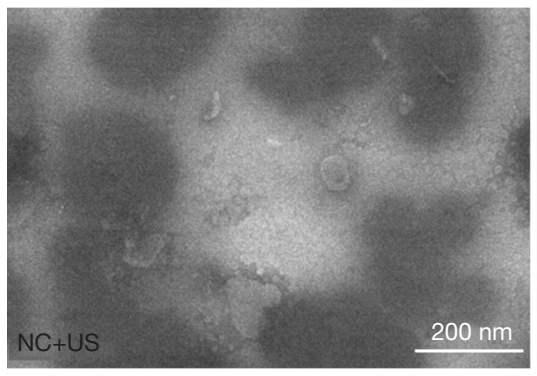

C

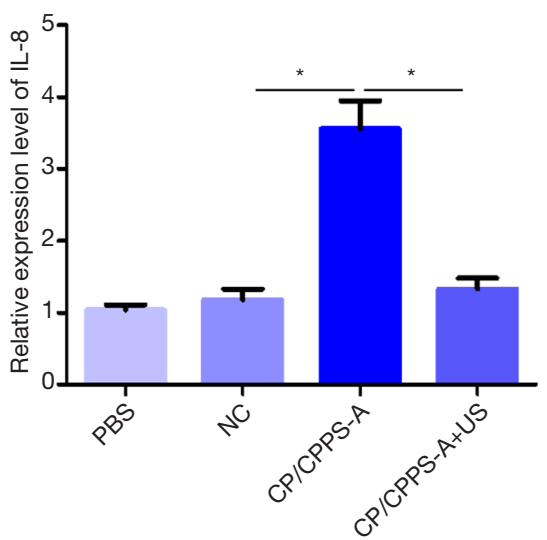

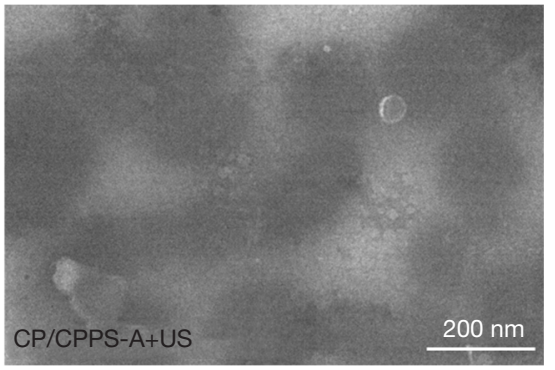
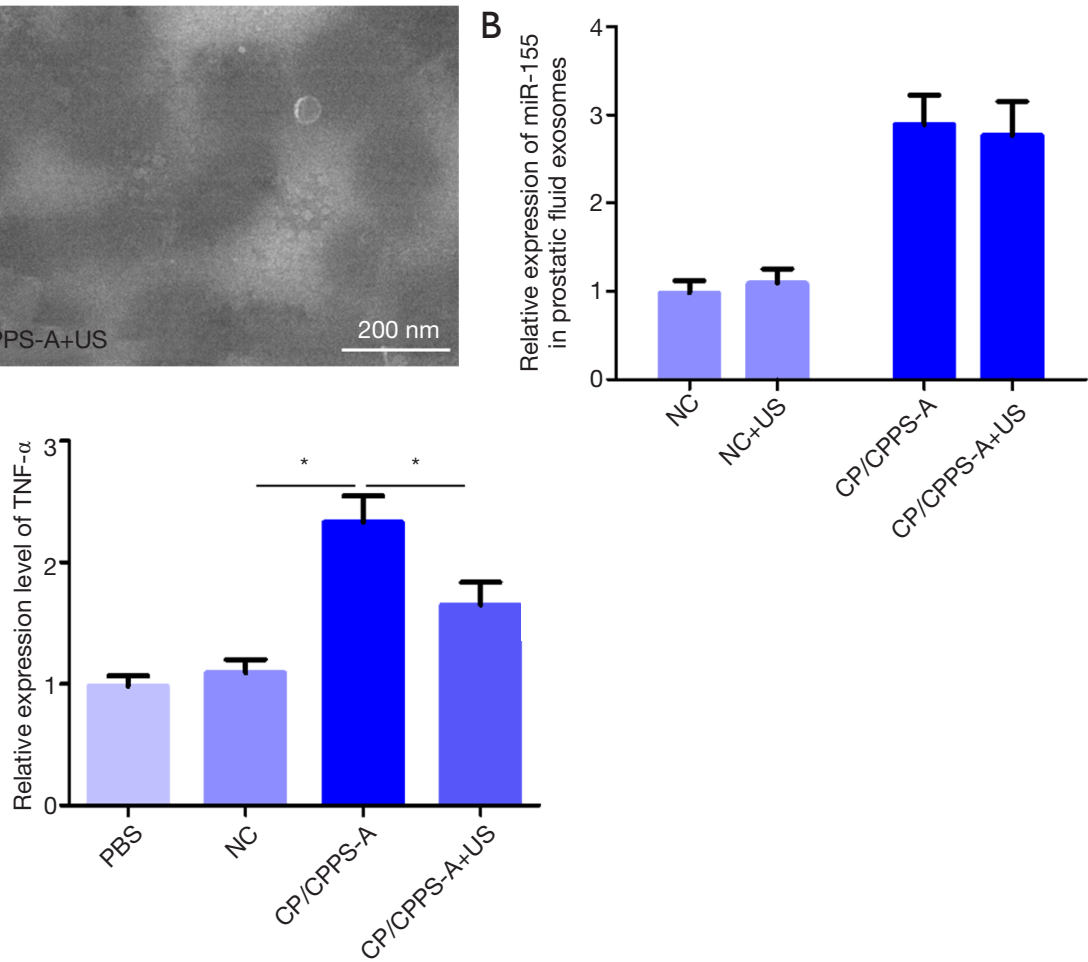

Figure 3 Prostatic fluid exosomes induced inflammation of prostatic stromal cells. (A) Representative images of transmission electron microscopy of prostatic fluid exosomes after ultrasound-rupture treatment extracted from a healthy individual and a CP/CPPS-A patient, scale bar $=200 \mathrm{~nm}$. (B) Relative miRNA-155 expression in the prostatic fluid exosomes of healthy individuals and CP/CPPS-A patients before and after the ultrasound-rupture treatment. (C,D) Relative IL-8 and TNF- $\alpha$ expression levels of exosomes co-cultured with prostatic stromal cells co-cultured in different groups detected by ELISA. *, P<0.05. PBS, phosphate buffer saline; NC, normal control; CP/CPPS-A, type IIIA chronic prostatitis/chronic pelvic pain syndrome; CP/CPPS-A + US, ultrasonic treatment in the CP/CPPS-A.

suggesting that miRNA-155 was selectively loaded into exosomes of CP/CPPS-A patients and was taken up by prostatic stromal cells.

\section{Prostatic fluid exosomes induce inflammation of prostatic stromal cells}

We performed ultrasonic treatment on prostatic fluid exosomes to destroy the exosomes' double-layer plasma membrane structure, followed by observation of the effect of ultrasonic treatment using a transmission electron microscope. Our results showed that after ultrasonic treatment, the intact exosome plasma membrane structure was rarely observed in both $\mathrm{NC}$ plus ultrasound-rupture treatment $(\mathrm{NC}+\mathrm{US}$ - prostatic fluid exosomes of healthy individuals with ultrasound-rupture treatment) and $\mathrm{CP} /$ CPPS-A + US groups by electron microscopy (Figure 3A), indicating that most of the exosome membranes were destroyed. Second, we retested the concentration of miRNA-155 in exosomes after ultrasonic treatment and showed that the miRNA-155 concentration did not change significantly with ultrasonic destruction of the exosomal membrane (NC vs. NC+ US and CP/CPPS-A vs. CP/ CPPS-A + US, Figure 3B).

To observe prostatic fluid exosomes' effect on prostatic stromal cells, we co-cultured the WPMY-1 cells with PBS and with different groups of exosomes (i.e., NC, CP/ CPPS-A, and CP/CPPS-A + US groups). After 48 h, we detected IL- 8 and TNF- $\alpha$ concentrations using ELISA. Our results showed that IL- 8 and TNF- $\alpha$ concentrations of the CP/CPPS-A group were significantly higher than those of the NC group. Also, the IL- 8 and TNF- $\alpha$ concentrations of exosomes with ultrasonic treatment in the CP/CPPS-A (CP/CPPS-A + US) group were significantly lower than those of the exosomes without ultrasonic treatment in the CP/CPPS-A group (Figure 3C and 3D). These results 
indicated that exosomes derived from prostatic fluid in the $\mathrm{CP} / \mathrm{CPPS}-\mathrm{A}$ patients induced inflammation, which was greatly weakened by ultrasonic treatment.

\section{Prostatic fluid exosomes promote inflammation of the prostate tissue}

In the animal experiments, we injected the same amount of normal saline or corresponding exosomes into the ventral lobe of the prostate of male SD rats in individual groups (i.e., NS, NC, CP/CPPS-A, and CP/CPPS-A + US). The animals were euthanized a week after to collect the prostate tissue for paraffin-embedding and sectioning. HE-staining results showed that sections of the exosomes injected with normal saline and normal control prostatic fluid had normal prostate morphology, intact acinar structures, and no obvious inflammatory-cell infiltration in the interstitial tissues. However, prostatic fluid exosomes and sections of prostatic fluid exosomes of the CP/CPPS-A patients treated with ultrasound had many inflammatory-cell infiltrations in the interstitial tissues. Importantly, the inflammatorycell infiltration in the ultrasonic treatment group was significantly improved compared with the untreated group (Figure 4A).

To further investigate the prostate tissue's inflammation, we used immunofluorescence staining to detect the expression of IL- 8 , TNF- $\alpha$, and iNOS in the prostate tissue. Our results showed that the above cytokines were not significantly expressed in the NS and NC groups but were very significantly expressed in the CP/CPPS-A group. Compared with the NC group, the CP/CPPS-A + US group had a higher expression of IL-8, TNF- $\alpha$, and iNOS, which was significantly lower than in the CP/CPPS-A group (Figure $4 B, C, D$ ).

\section{miRNA-155 promoted inflammation of prostatic stromal cells}

Considering the prostatitis effect of exosomes derived from prostatic fluid in patients with CP/CPPS-A and the significant upregulation of $m i R N A-155$, we verified whether miRNA-155 could mimic the inflammatory response induced by exosomes using commercially available $m i R N A-155$ inhibitor or mimic and negative control inhibitor or mimic to perform miRNA functional analysis. After transfection of prostatic stromal cells, the results showed that $50 \mathrm{nM} m i R N A-155$ mimic significantly enhanced the expression of miRNA-155, and $100 \mathrm{nM}$
miRNA-155 inhibitor significantly reduced the expression of miRNA-155 (Figure $5 A$ ).

We used ELISA to detect the various inflammatory cytokines in the transfected prostatic stromal cells. The enhanced expression of miRNA-155 increased IL-8 and TNF- $\alpha$ expression. Also, IL- 8 and TNF- $\alpha$ expression did not change significantly after inhibiting miRNA-155 expression (Figure 5B,C), suggesting that high miRNA-155 expression promoted inflammation prostatic stromal cells, whereas low miRNA-155 expression did not have an inflammatory protective effect.

\section{Discussion}

Although many studies have been conducted on prostatitis, traditional research ideas on inflammation have not clarified the pathogenic mechanisms of prostatitis. Increasing evidence has shown that the main role of exosomes is not as a carrier for cell excretion but rather as a key signal carrier in the process of short- and long-distance cell signaling (26). As mentioned in the introduction, exosomes are secreted into various body fluids, including prostatic fluid and urine. Many studies have suggested that exosomes correlate strongly with certain chronic inflammatory diseases (12-15). We speculate that exosomes and their carried contents may play an important role in the occurrence and progression of CP/CPPS-A.

In this study, we successfully extracted and identified exosomes from human prostatic fluid and found a significant difference in the concentrations of prostatic fluid exosomes between CP/CPPS-A patients and healthy individuals. Histologically, prostate epithelial cells and prostatic stromal cells are adjacent to each other, creating conditions for exosomes to pass between these two types of cells. Also, many researchers believe that prostatic stromal cells are immunogenic in their studies (24). To further verify whether prostatic fluid exosomes were involved in initiating prostatitis, we used the PKH26 dye to label the prostatic fluid-derived exosomes and co-cultured labeled exosomes with human prostatic stromal cells. Interestingly, most of these exosomes easily entered the prostatic stromal cells. These results will aid subsequent functional analysis of exosomes.

Most of the existing studies have shown that exosomes participate in the pathophysiological process of various diseases through paracrine and remote secretion as a carrier of signaling molecules. Recently, it was reported in highprofile journals that exosomes are used as carriers to mediate 

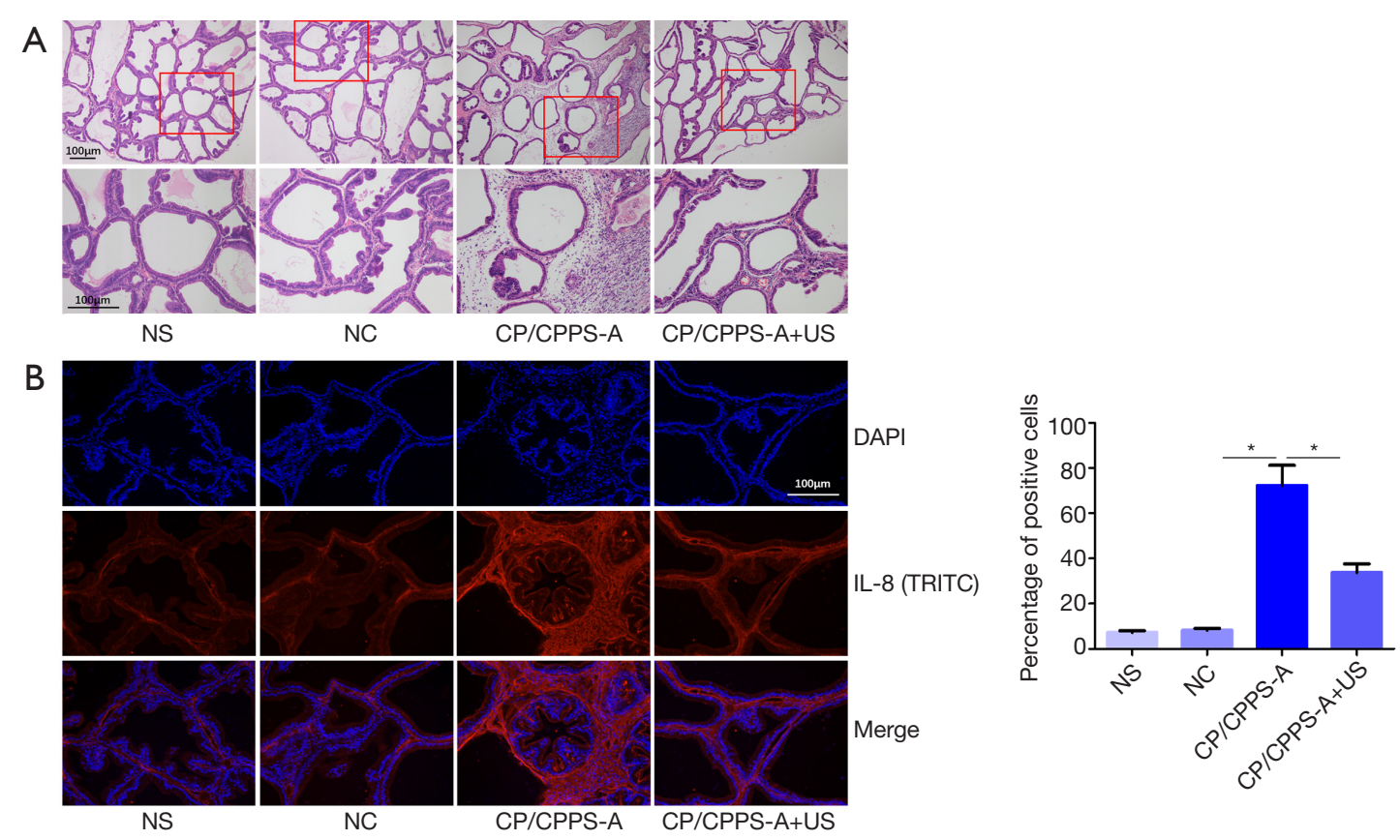

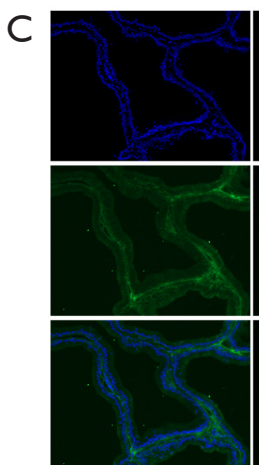

NS

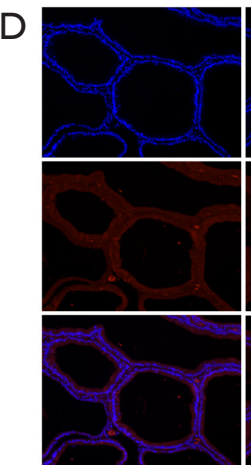

NS

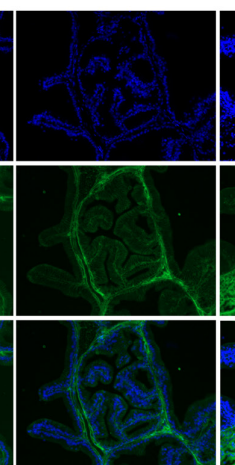

NC

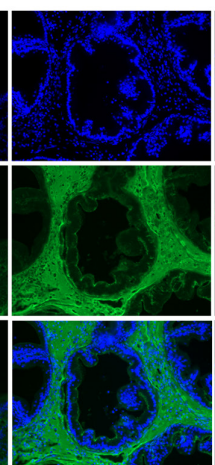

CP/CPPS-A

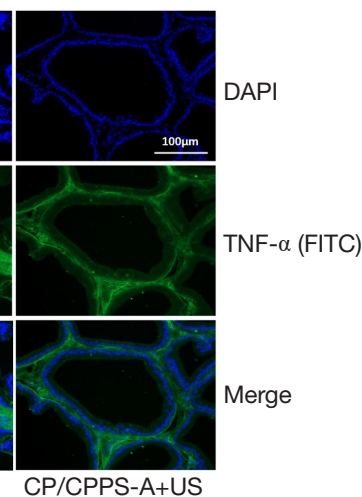

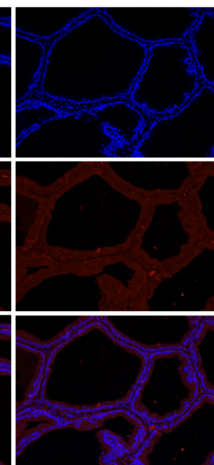

NC

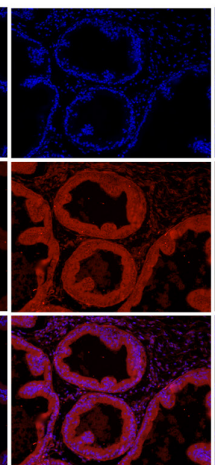

CP/CPPS-A

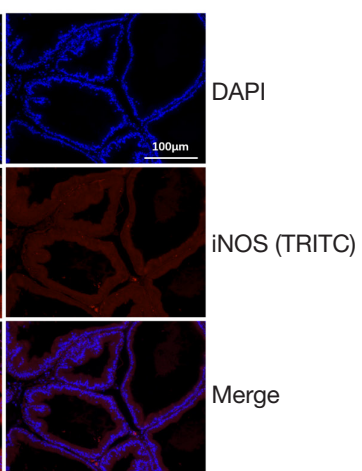

CP/CPPS-A+US
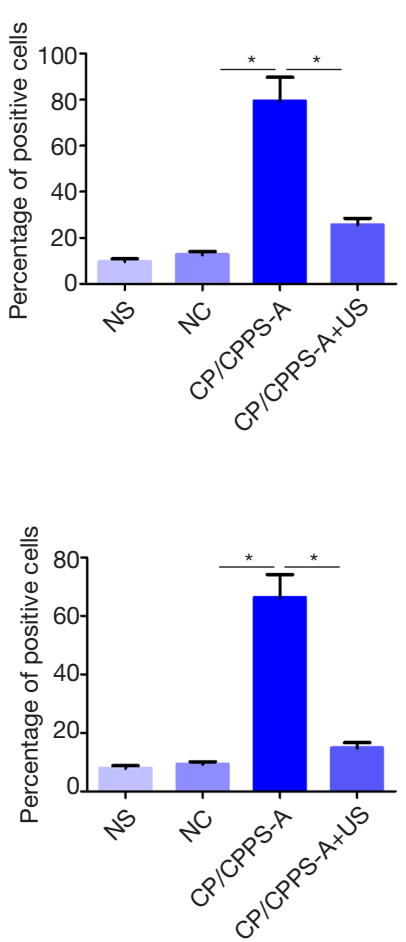

Figure 4 Figure 4 Prostatic fluid exosomes promoted inflammation in prostate tissue. (A) Representative HE-staining images of prostate tissue in SD rats injected with normal saline, NC exosomes, prostatic fluid exosomes of CP/CPPS-A patients, and ultrasound-treated prostatic fluid exosomes of CP/CPPS-A patients. (B) Results of prostate tissue in different groups measured by MPO activity assay. (C-E) Expression of IL-8 (TRITC secondary antibodies), TNF- $\alpha$ (FITC secondary antibodies), and iNOS (TRITC secondary antibodies) in different groups of prostate tissue observed under a fluorescence microscope, scale bar $=100 \mu \mathrm{m}$. * $\mathrm{P}<0.05$. NS, normal saline; NC, normal control; CP/CPPS-A, type IIIA chronic prostatitis/chronic pelvic pain syndrome; CP/CPPS-A + US, ultrasonic treatment in the CP/CPPS-A; MPO, myeloperoxidase. 
A

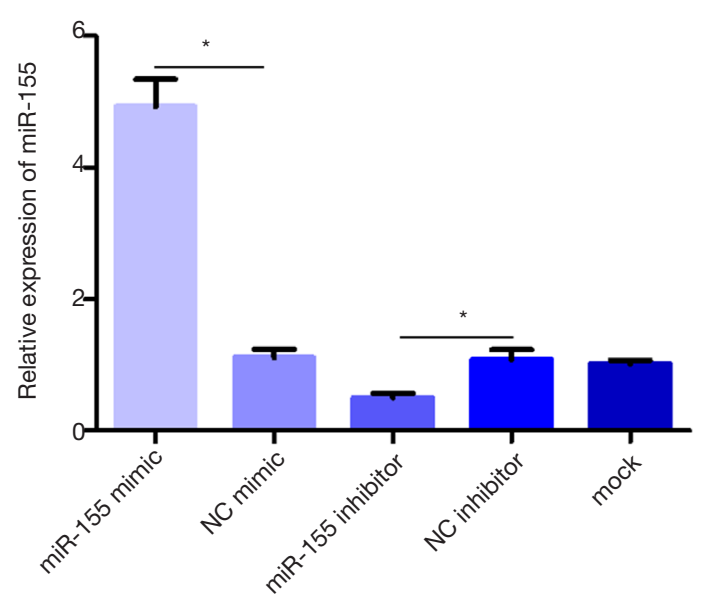

B

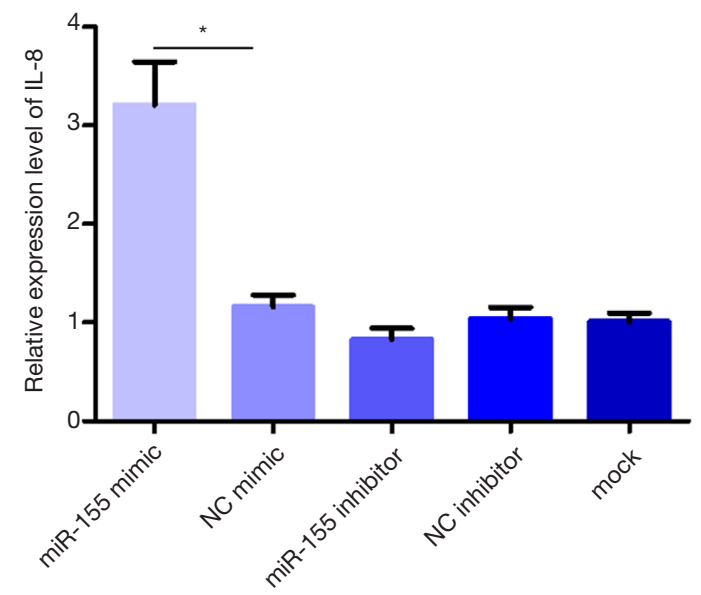

C

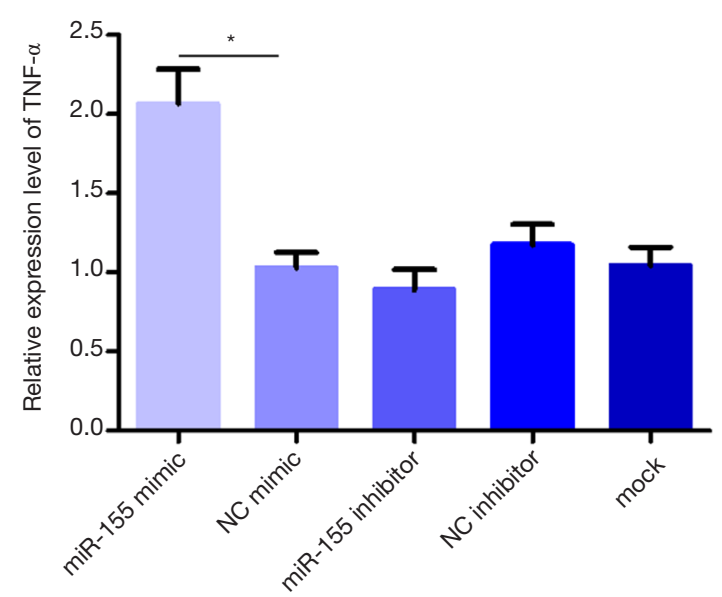

Figure 5 miRNA-155 promoted inflammation of prostatic stromal cells. (A) Expression of $m i R N A-155$ in prostatic stromal cells transfected with $m i R-155$ inhibitor/mimic and negative control inhibitor/mimic measured by qPCR. (B,C) ELISA sequentially measured the relative expression levels of IL-8 and TNF- $\alpha$ in the prostatic stromal cell culture system after regulating the expression of miRNA-155. *, $\mathrm{P}<0.05$. specific miRNA transfer between cells and play a key role in the occurrence and progression of decreases, such as lung injury, diabetes, and vascular dysplasia $(22,27,28)$. Regarding inflammation, exosomes secreted by neutrophils deliver $m i R N A-155$ to vascular endothelial cells to initiate atherosclerosis and the progression of chronic vascular inflammation (13). For refractory temporomandibular joint osteoarthritis, exosomes secreted by mesenchymal stem cells reduce inflammation and restore matrix homeostasis (15).

miRNA-155 is a highly conserved and important miRNA and has been considered to correlate strongly with inflammatory diseases. Importantly, a recent study has shown that miRNA-155 may be related to the occurrence of prostatitis, and changes in the level of miRNA-155 affect the inflammatory response and oxidative stress of autoimmune prostatitis (29), which arouse further interest in the expression of $m i R N A-155$ in exosomes. In this study, after testing different groups of exosomes, we showed that the concentration of miRNA-155 in exosomes derived from prostatic fluid in CP/CPPS-A patients was abnormally increased, suggesting that miRNA-155 was selectively loaded into exosomes and was transported to prostatic stromal cells. These results were consistent with the highthroughput sequencing results of miRNAs in the prostatic fluid of patients with chronic prostatitis reported previously. This study also tested the inflammatory cytokines on the coculture prostatic stromal cell line and showed that exosomes derived from prostatic fluid in CP/CPPS-A patients promoted the expression of IL- 8 and TNF- $\alpha$. According to previous reports and our results, we believe that exosomes containing high concentrations of $m i R N A-155$ were a prostatitis-promoting factor.

Exosomes, as macromolecular substances, enter and exit the cell membrane through endocytosis and exocytosis, which require the integrity of the double-layer plasma membrane structure. A previous study has shown that high-frequency ultrasound destroys the plasma membrane structure of exosomes (30), which suggests ways of seeking interventions for pro-inflammatory targets of exosomes. This study used high-frequency ultrasound to process the exosomes derived from the prostatic fluid of CP/CPPS-A patients and used transmission electron microscopy to show that ultrasound treatment destroys most of the exosomal plasma membrane structure. However, the detection of miRNA-155 did not show any obvious reduction in the miRNA level, indicating that ultrasound only disrupted the exosomal plasma membrane structure but did not affect the exosomes' miRNA-155 concentration. In further 
in vitro experiments, we showed that the pro-inflammatory ability of prostatic fluid exosomes in CP/CPPS-A patients treated with ultrasound was weakened, as manifested by the decreased inflammatory cytokine expression. In in vivo experiments, immunofluorescence staining was used to detect the expression of inflammatory cytokines more intuitively in the prostatic fluid exosomes of CP/CPPS-A patients after ultrasound-rupture treatment. The results showed that the expression of inflammatory cytokines in the prostatic fluid exosomes of the CP/CPPS-A + US group was lower than that of the CP/CPPS-A group. The positive expression of IL-8 and TNF- $\alpha$ was mainly localized in the prostate's stroma and mesenchyme, and the positive expression of iNOS was mainly localized in the prostate epithelial cells.

To eliminate the potential interference caused by other substances in exosomes and verify whether miRNA-155 mimicked exosome-induced prostatitis, we conducted functional analyses of $m i R N A-155$. Our results showed that the reduction of $m i R N A-155$ did not affect IL- 8 or TNF- $\alpha$ expression, while simultaneous increases in IL-8 and TNF- $\alpha$ were observed after increasing miRNA-155. These results further proved the important role of high miRNA-155 concentrations in the occurrence and progression of prostatitis.

\section{Conclusions}

In this study showed that there were abnormally high concentrations of exosomes in the prostatic fluid of patients with CP/CPPS-A. These exosomes were selectively loaded with miRNA-155 and were engulfed by prostatic stromal cells in large quantities to activate the immune response and induce inflammation. These results demonstrate a new mechanism in which inflammation may be stimulated in CPPS patients who have WBC in their prostatic fluid. Further study of the signal transduction pathways on which the pro-inflammatory effects of exosomes depend is necessary.

\section{Acknowledgments}

We thank LetPub (www.letpub.com) for its linguistic assistance during the preparation of this manuscript.

Funding: None.

\section{Footnote}

Reporting Checklist: The authors have completed the MDAR reporting checklist. Available at http://dx.doi.org/10.21037/ tau-21-139

Data Sharing Statement: Available at http://dx.doi. org/10.21037/tau-21-139

Conflicts of Interest: All authors have completed the ICMJE uniform disclosure form (available at http://dx.doi. org/10.21037/tau-21-139). The authors have no conflicts of interest to declare.

Ethical Statement: The authors are accountable for all aspects of the work in ensuring that questions related to the accuracy or integrity of any part of the work are appropriately investigated and resolved. All volunteers included in the study provided written informed consent before participating in this study. The research protocol was reviewed and approved by the Ethics Committee of the First Affiliated Hospital of Army Medical University (Ethics approval number: KY201801). The study was conducted in accordance with the Declaration of Helsinki (as revised in 2013).

Open Access Statement: This is an Open Access article distributed in accordance with the Creative Commons Attribution-NonCommercial-NoDerivs 4.0 International License (CC BY-NC-ND 4.0), which permits the noncommercial replication and distribution of the article with the strict proviso that no changes or edits are made and the original work is properly cited (including links to both the formal publication through the relevant DOI and the license). See: https://creativecommons.org/licenses/by-nc-nd/4.0/.

\section{References}

1. Anothaisintawee T, Attia J, Nickel JC, et al. Management of chronic prostatitis/chronic pelvic pain syndrome: a systematic review and network meta-analysis. JAMA 2011;305:78-86.

2. Franco JV, Turk T, Jung JH, et al. Pharmacological interventions for treating chronic prostatitis/chronic pelvic pain syndrome. Cochrane Database Syst Rev 2019;10:CD012552.

3. Lai HH, Thu JHL, Moh FV, et al. Clustering of Patients with Interstitial Cystitis/Bladder Pain Syndrome and Chronic Prostatitis/Chronic Pelvic Pain Syndrome. J Urol 2019;202:546-51.

4. Khan FU, Ihsan AU, Khan HU, et al. Comprehensive 
overview of prostatitis. Biomed Pharmacother 2017;94:1064-76.

5. Doyle LM, Wang MZ. Overview of Extracellular Vesicles, Their Origin, Composition, Purpose, and Methods for Exosome Isolation and Analysis. Cells 2019;8:727.

6. Mathieu M, Martin-Jaular L, Lavieu G, et al. Specificities of secretion and uptake of exosomes and other extracellular vesicles for cell-to-cell communication. Nat Cell Biol 2019;21:9-17.

7. Pegtel DM, Gould SJ. Exosomes. Annu Rev Biochem 2019;88:487-514.

8. Théry C, Zitvogel L, Amigorena S. Exosomes: composition, biogenesis and function. Nat Rev Immunol 2002;2:569-79.

9. Yan W, Jiang S. Immune Cell-Derived Exosomes in the Cancer-Immunity Cycle. Trends Cancer 2020;6:506-17.

10. Barile L, Vassalli G. Exosomes: Therapy delivery tools and biomarkers of diseases. Pharmacol Ther 2017;174:63-78.

11. Patil M, Henderson J, Luong H, et al. The Art of Intercellular Wireless Communications: Exosomes in Heart Disease and Therapy. Front Cell Dev Biol 2019;7:315.

12. Bandopadhyay M, Sarkar N, Datta S, et al. Hepatitis B virus $\mathrm{X}$ protein mediated suppression of miRNA-122 expression enhances hepatoblastoma cell proliferation through cyclin G1-p53 axis. Infect Agent Cancer 2016;11:40.

13. Gomez I, Ward B, Souilhol C, et al. Neutrophil microvesicles drive atherosclerosis by delivering miR-155 to atheroprone endothelium. Nat Commun 2020;11:214.

14. Jiang M, Fang H, Shao S, et al. Keratinocyte exosomes activate neutrophils and enhance skin inflammation in psoriasis. FASEB J 2019;33:13241-53.

15. Zhang S, Teo KYW, Chuah SJ, et al. MSC exosomes alleviate temporomandibular joint osteoarthritis by attenuating inflammation and restoring matrix homeostasis. Biomaterials 2019;200:35-47.

16. Hanna J, Hossain GS, Kocerha J. The Potential for microRNA Therapeutics and Clinical Research. Front Genet 2019;10:478.

17. Sheikh Hassani M, Green JR. Multi-view Co-training for microRNA Prediction. Sci Rep 2019;9:10931.

18. Dai R, Ahmed SA. MicroRNA, a new paradigm for understanding immunoregulation, inflammation, and autoimmune diseases. Transl Res 2011;157:163-79.

19. Hayashi T, Hoffman MP. Exosomal microRNA communication between tissues during organogenesis. Rna Biology 2017;14:1683-9.
20. Kamity R, Sharma S, Hanna N. MicroRNA-Mediated Control of Inflammation and Tolerance in Pregnancy. Front Immunol 2019;10:718.

21. Cardoso AL, Guedes JR, Pereira de Almeida L, et al. miR155 modulates microglia-mediated immune response by down-regulating SOCS-1 and promoting cytokine and nitric oxide production. Immunology 2012;135:73-88.

22. Jiang K, Yang J, Guo S, et al. Peripheral Circulating Exosome-Mediated Delivery of miR-155 as a Novel Mechanism for Acute Lung Inflammation. Molecular Therapy 2019;27:1758-71.

23. Zhang L, Liu Y, Chen XG, et al. MicroRNA expression profile in chronic nonbacterial prostatitis revealed by next-generation small RNA sequencing. Asian J Androl 2019;21:351-9.

24. Wang R, Zhang M, Ou Z, et al. Long noncoding RNA DNM3OS promotes prostate stromal cells transformation via the miR-29a/29b/COL3A1 and miR-361/TGFbeta1 axes. Aging (Albany NY) 2019;11:9442-60.

25. Genschmer KR, Russell DW, Lal C, et al. Activated PMN Exosomes: Pathogenic Entities Causing Matrix Destruction and Disease in the Lung. Cell 2019;176:11326.e15.

26. Kalluri R, LeBleu VS. The biology, function, and biomedical applications of exosomes. Science 2020;367:eaau6977.

27. Xu B, Zhang Y, Du XF, et al. Neurons secrete miR-132containing exosomes to regulate brain vascular integrity. Cell Research 2017;27:882-97.

28. Ying $W$, Riopel $M$, Bandyopadhyay $G$, et al. Adipose Tissue Macrophage-Derived Exosomal miRNAs Can Modulate In Vivo and In Vitro Insulin Sensitivity. Cell 2017;171:372-84.e12.

29. Fu X, He HD, Li CJ, et al. MicroRNA-155 deficiency attenuates inflammation and oxidative stress in experimental autoimmune prostatitis in a TLR4dependent manner. Kaohsiung J Med Sci 2020;36:712-20.

30. Lee JY, Park JK, Lee EY, et al. Circulating exosomes from patients with systemic lupus erythematosus induce an proinflammatory immune response. Arthritis Res Ther 2016;18:264. Erratum in: Arthritis Res Ther 2020;22:109.

Cite this article as: Zhao B, Zheng J, Qiao Y, Wang Y, Luo Y, Zhang D, Cai Q, Xu Y, Zhou Z, Shen W. Prostatic fluid exosome-mediated microRNA-155 promotes the pathogenesis of type IIIA chronic prostatitis. Transl Androl Urol 2021;10(5):1976-1987. doi: 10.21037/tau-21-139 